\title{
Tritium in Mondproben
}

von P. Bochsler, P. Eberhardt, J. Geiss, H. Loosli, H. Oeschger und M. Wahlen (Physikalisches Institut der Universität Bern)

Siehe: Tritium in Lunar Material, Proc. Apollo 12 Lunar Science Conference, Geochim. Cosmochim. Acta, Suppl. II (1971), in press.

\section{Strahlungsalter und Bestrahlungsgeschichte von Apollo-11- und -12-Steinen}

von P. Eberhardt, J. Geiss, H. Graf, N. Grögler, U. Krähenbühl, H. Schwaller, J. Schwarzmüller und A. Stettler

(Physikalisches Institut der Universität Bern)

Siehe: Correlation between Rock Type and Irradiation History of Apollo 11 Igneous Rocks, Earth and Planetary Science Letters 10, 67-72 (1970).

\section{Edelgase des Sonnenwinds in einem Aluminiumrohr von Surveyor 3}

von F. Bühler, P. Eberhardt, J. Geiss und J. SchwarzmülleR

(Physikalisches Institut der Universität Bern)

Siehe: Trapped Solar Wind Helium and Neon in Surveyor 3 Material, Earth and Planetary Science Letters 10, 297-306 (1971).

\section{Messtechnische Anwendungen von Gyratorkreisen \\ von P. Vogel und E. BALDINGER \\ (Institut für angewandte Physik der Universität Basel)}

Gyratoren sind elektrische Vierpole, welche mit Widerständen und Kondensatoren zusammen die Synthese von Netzwerken mit komplexen Polpaaren gestatten. Gyratorkreise können auch für Messungen von Phasenverschiebungen bei Vierpolen und zu Verlustwinkelmessungen an Kondensatoren verwendet werden. Es wird über die Anwendung von Gyratorkreisen bei der Untersuchung der Phasengänge aktiver Elemente für aktive RC-Kreise berichtet.

\section{Cinétique d'adsorption des centres catalytiques sur le $\mathrm{TiO}_{2}$ \\ par Charles Hauser \\ (Laboratoire d'Optique physique EPF, Lausanne)}

L'adsorption d'oxygène sur de l'oxyde de titane partiellement désoxydé en surface fait apparaître un signal paramagnétique composé de deux raies de trois valeurs de $g$ chacune.

L'étude de ces cinétiques montre qu'en fin de réaction la variation est exponentielle, alors qu'au début un fort écart est enregistré. Nous avons été amenés à penser 